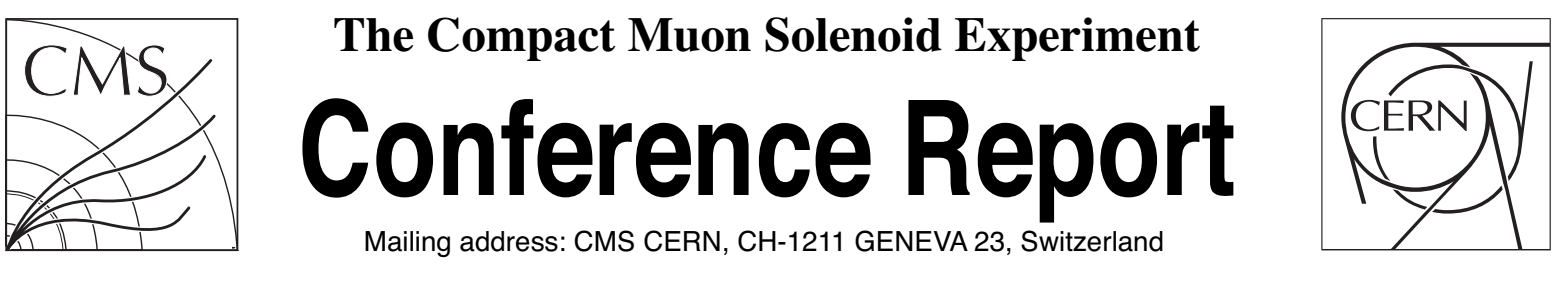

03 October 2016 (v2, 06 October 2016)

\title{
The CT-PPS tracking system with 3D pixel detectors
}

Fabio Ravera for the CMS Collaboration

\begin{abstract}
The CMS-TOTEM Precision Proton Spectrometer (CT-PPS) detector will be installed in Roman pots (RP) positioned on either side of CMS, at about $200 \mathrm{~m}$ from the interaction point. This detector will measure forward leading protons, allowing detailed studies of diffractive physics and central exclusive production in standard LHC running conditions. An essential component of the CT-PPS apparatus is the tracking system, which consists of two detector stations per arm equipped with six 3D silicon pixelsensor modules, each read out by six PSI46dig chips. The front-end electronics has been designed to fulfill the mechanical constrains of the RP and to be compatible as much as possible with the readout chain of the CMS pixel detector. The tracking system is currently under construction and will be installed by the end of 2016. In this contribution the final design and the expected performance of the CT-PPS tracking system will be presented. A summary of the studies performed, before and after irradiation, on the 3D detectors produced for CT-PPS will be given.
\end{abstract}




\title{
The CT-PPS tracking system with 3D pixel detectors
}

\author{
F. Ravera, ${ }^{a, 1}$ on behalf of the CMS and TOTEM Collaborations \\ ${ }^{a}$ University of Torino and INFN, Torino, Italy \\ E-mail: fabio.ravera@cern.ch
}

AbstRact: The CMS-TOTEM Precision Proton Spectrometer (CT-PPS) detector will be installed in Roman pots (RP) positioned on either side of CMS, at about $200 \mathrm{~m}$ from the interaction point. This detector will measure leading protons, allowing detailed studies of diffractive physics and central exclusive production in standard LHC running conditions. An essential component of the CT-PPS apparatus is the tracking system, which consists of two detector stations per arm equipped with six 3D silicon pixel-sensor modules, each read out by six PSI46dig chips. The front-end electronics has been designed to fulfill the mechanical constraints of the RP and to be compatible as much as possible with the readout chain of the CMS pixel detector. The tracking system is currently under construction and will be installed by the end of 2016. In this contribution the final design and the expected performance of the CT-PPS tracking system is presented. A summary of the studies performed, before and after irradiation, on the 3D detectors produced for CT-PPS is given.

KeYwords: Solid state detectors, Radiation-hard detectors, Particle tracking detectors, Hybrid detectors, Front-end electronics for detector readout

\footnotetext{
${ }^{1}$ Corresponding author.
} 


\section{Contents}

1 Introduction 1

2 3D Pixel detector 2

2.1 CMN wafer production 2

2.2 Beam tests of CNM single-ROC sensors 3

2.3 CT-PPS module qualification 5

3 CT-PPS tracking electronics, mechanics and cooling 5

4 Conclusions $\quad 6$

\section{Introduction}

The CMS-TOTEM Precision Proton Spectrometer (CT-PPS) will allow the study of central exclusive production in proton-proton collisions, namely the process $p p \rightarrow p X p$ [1]. The spectrometer will be installed on both sides of the CMS apparatus [2] at $200 \mathrm{~m}$ from the Interaction Point (IP), taking advantage of the LHC magnets to bend the scattered protons.

The CT-PPS detector consists of a silicon tracking system, which is the main subject of this paper, to measure the position and direction of the protons, and a timing detector to measure their arrival time. This will allow to reconstruct momentum and mass of the centrally produced system $X$, as well as its primary vertex. Both tracking and timing detectors will be installed along the LHC beam-pipe inside Roman Pots (RP) that can be moved very close to the beam.

Because of the interest in centrally produced resonances decaying into diphotons raised by the CMS and ATLAS results [4, 5], TOTEM silicon strip detectors were installed in the CT-PPS RP for the 2016 data taking. During the 2016-17 winter shutdown they will be replaced by pixel detectors, which will better sustain pile-up during standard high-luminosity fills.

Because of the small distance from the beam of the CT-PPS detectors, the silicon modules are foreseen to face an irradiation of $5 \times 10^{15}$ proton $/ \mathrm{cm}^{2}$ for $100 \mathrm{fb}^{-1}$ of collected luminosity. This corresponds to a fluence between 1 and $3 \times 10^{15} \mathrm{n}_{\mathrm{eq}} / \mathrm{cm}^{2}$. Besides the radiation hardness, another requirement is the reduction of the insensitive area at the edge of the sensor, in order to increase the acceptance for high-momentum protons. Finally, a tracking resolution of $\sim 10 \mu \mathrm{m}$ on both $x$ and $y$ directions is necessary to measure with good resolution the proton kinematic variables. The chosen design is to use six planes of $3 \mathrm{D}$ pixel detectors for each station, tilted by $18.4^{\circ}$ to increase the resolution. 


\section{3D Pixel detector}

The main feature of 3D sensors is that, instead of implementing the electrodes on the surfaces, as in the planar technology, they are etched in a column shape perpendicular to the surface, thus decoupling the sensor thickness from the distance between electrodes. The main advantages are low depletion voltage and high signal collection efficiency after irradiation.

3D pixel detectors have been chosen because of their intrinsic radiation hardness and the possibility to implement slim edges. Several test-beam campaigns on sensors from different vendors - CNM (Barcelona, Spain), FBK (Trento, Italy) and SINTEF (Oslo, Norway) - have been carried out, with results that confirmed the suitability of this technology for CT-PPS. The order was eventually placed with CNM.

\subsection{CMN wafer production}

The CNM 3D production is double-sided with not-passing-through columns, meaning that $n^{+}$ and $p^{+}$electrodes are etched from opposite surfaces of the wafer and they stop before reaching the other surface. A sketch is shown in Fig. 1a.

The CT-PPS modules are read out with the PSI46dig ReadOut Chip (ROC), the same used for the CMS Phase I pixel detector [3]. The readout chip is made of a $52 \times 80$ matrix of pixels with size $150 \times 100 \mu \mathrm{m}^{2}$.

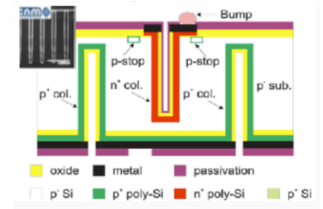

(a). CNM 3D cross section. $1 \mathrm{E}$

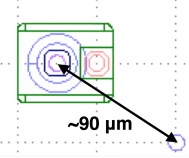

(b). 1E pixel layout.

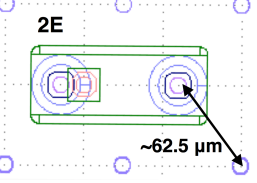

(c). 2E pixel layout.

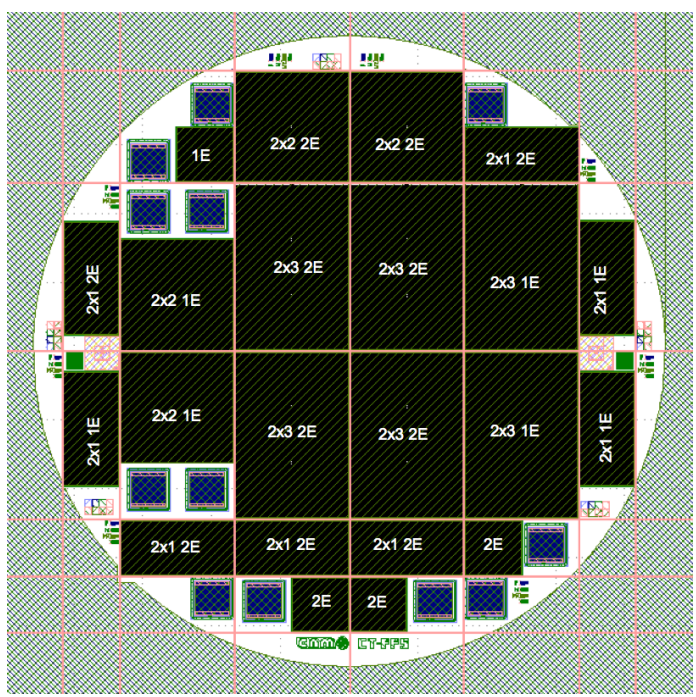

(d). Wafer layout for the CT-PPS 3D sensors produced at CNM.

Figure 1. Characteristics of the CNM 3D sensor production for CT-PPS.

Two different pixel layouts have been chosen for the CT-PPS production, with one and two readout columns, referred as $1 \mathrm{E}$ and $2 \mathrm{E}$, respectively. 2E sensors (Fig. 1c) have a shorter interelectrode distance with respect to $1 \mathrm{E}$ ones (Fig. 1b), ensuring better performance after irradiation, but with an expected lower yield because of the higher column density. Therefore $1 \mathrm{E}$ sensors were also included in the wafer layout (Fig. 1d) as backup. Sensors have a $200 \mu \mathrm{m}$ slim edge made with a p-doped column fence. 
The module dimension is expressed in terms of the number of bump-bonded ROCs. The baseline detector concept uses $2 \times 3$ sensors, which means readout by 6 ROCs, but the electronics is compatible with $2 \times 2$ modules also. Each wafer contains $2 \times 3$ and $2 \times 2$ sensors for the module production, together with $2 \times 1$, single ROCs and diodes for testing purposes.

Sensors are required to have a breakdown voltage higher than $35 \mathrm{~V}$ and they are sub-divided in class $\mathrm{A}, \mathrm{B}$ and $\mathrm{C}$ based on the leakage current measured on the wafer at $20 \mathrm{~V}$ and room temperature by means of a temporary metal deposition. Class $\mathrm{C}$ sensors are rejected.

Two batches of twelve wafers each were produced. The first one was completed in December 2015 with good quality sensors, but low class A yield. The second one, finished in May 2016, had too low breakdown voltage. A low-dose neutron irradiation $\left(5-10 \times 10^{13} \mathrm{n}_{\mathrm{eq}} / \mathrm{cm}^{2}\right)$ is under investigation as a means to recover the production.

\subsection{Beam tests of CNM single-ROC sensors}

In March 201610 single-ROC sensors, 2 1E and 8 2E, were tested on beam at Fermilab. Sensors of both class A and B were chosen. The test beam is a $120 \mathrm{GeV}$ proton beam and the tracking system is composed of 8 planes of CMS pixel modules, 4 upstream and 4 downstream with respect to the position of the device-under-test. The achieved track resolution is $8 \mu \mathrm{m}$ in both $\mathrm{x}$ and $\mathrm{y}$ directions.

The efficiency as a function of the bias voltage (Fig. 2a) shows a very low depletion voltage, 3 (5) $\mathrm{V}$ for $2 \mathrm{E}$ (1E) sensors. Very important is also that from the efficiency point of view, no difference is found between class A and B sensors. The efficiency does not increase as a function of the angle between the sensor and the beam direction (Fig. 2b) since the not-fully-passing-through columns give already high efficiency at $0^{\circ}$. At $20^{\circ}$, a tilting angle close to the one in CT-PPS, an efficiency higher than $99.4 \%$ is achieved before irradiation.

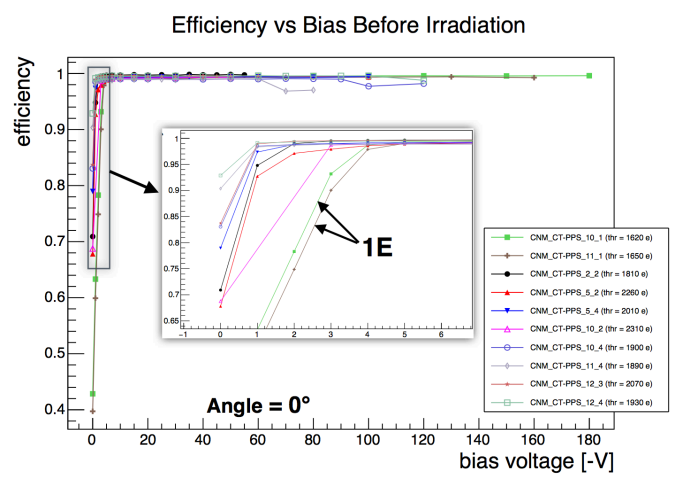

(a) Efficiency vs bias voltage.

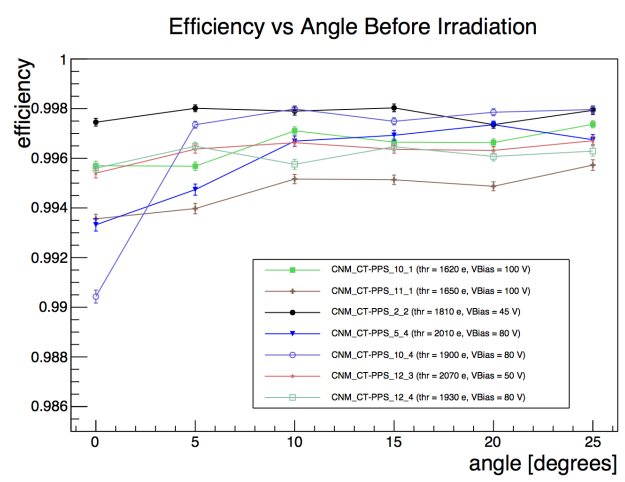

(b) Efficiency vs tilting angle.

Figure 2. Efficiency before irradiation as a function of bias voltage and tilting angle for single-ROC sensors from the first CT-PPS batch.

Another key point for CT-PPS is the reduction of the insensitive edge of the sensor. To evaluate it, the efficiency along the coordinate perpendicular to the sensor edge is measured for pixels at the border. Fitting the plot with a S-curve, as shown in Fig. 3a, it is possible to evaluate how much the efficiency extends beyond the geometrical edge of the pixel. If this value is plotted as a function of 
the bias voltage (Fig. 3b), it is possible to see how far the depletion region extends. Specifically, at $40 \mathrm{~V}, \sim 150 \mu \mathrm{m}(\sim 90 \mu \mathrm{m})$ of the $200 \mu \mathrm{m}$ slim edge can be recovered for $2 \mathrm{E}(1 \mathrm{E})$ detectors .

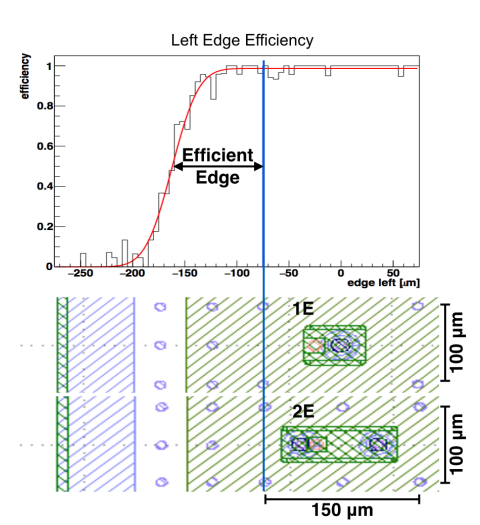

(a) Efficiency at the sensor edge.

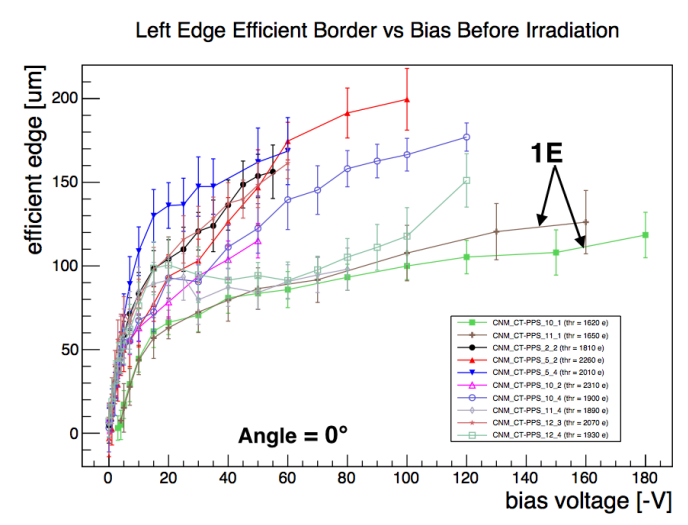

(b) Efficiency edge extension vs bias voltage.

Figure 3. Left plot: Efficiency along the coordinate perpendicular to the sensor edge for the pixel at the sensor border. The blue vertical line indicates the geometrical edge of the pixel, while the sensor cutting edge corresponds to the left edge of the histogram. Right plot: Efficiency extension as a function of the bias voltage.

Another important parameter for a tracking detector is the resolution. The residuals for pixel clusters of size one and two are measured and the telescope resolution is subtracted. To provide an average value, the resolution of the two cluster classes is weighted by the cluster size 2 probability shown in Fig. 4a. The sensor rotation with respect to the beam is around the same axis as in the final CT-PPS setup (y-axis in our reference system). The resulting resolution along $\mathrm{x}$ is shown in Fig. $4 \mathrm{~b}$ as a function of the tilting angle. As expected the resolution improves with the angle and reaches $\sim 22(25) \mu \mathrm{m}$ for $2 \mathrm{E}(1 \mathrm{E})$ sensors at $20^{\circ}$. Considering that 6 planes will be used for each station, the required $10 \mu \mathrm{m}$ resolution can be achieved.

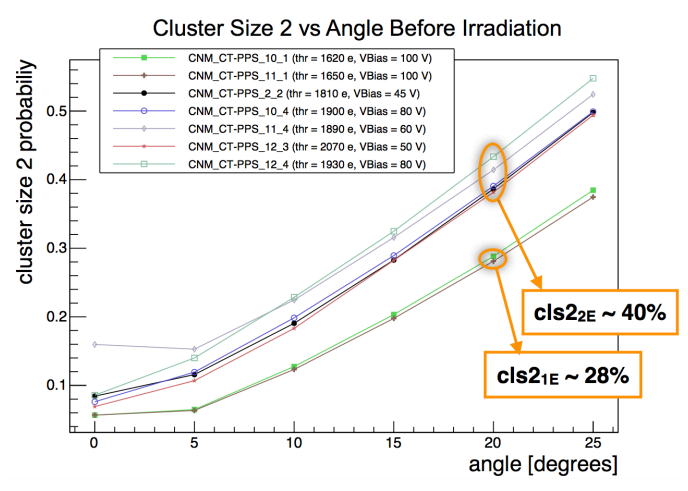

(a) Cluster size 2 probability vs tilting angle.

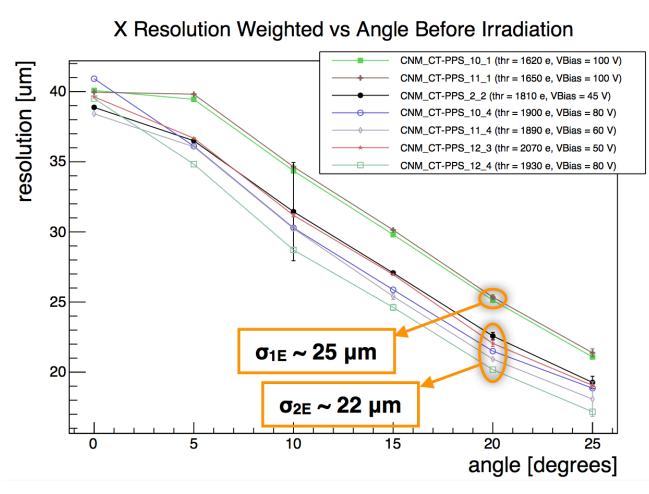

(b) X Resolution vs tilting angle.

Figure 4. Cluster size 2 probability as a function of the tilting angle (left plot). This is used for weighting cluster size 1 and 2 resolutions and estimate the global value (right plot).

Beam tests have also been done after irradiation. In this case, 1E CMS detectors from CNM 
ATLAS IBL wafers were used, plus a 2E SINTEF sensor since 2E CNM ones were not available at the time of the irradiation campaign. Sensors have been irradiated to fluences of 1 and $3 \times$ $10^{15} \mathrm{n}_{\mathrm{eq}} / \mathrm{cm}^{2}$ at the CERN IRRAD Proton Facility with $24 \mathrm{GeV}$ protons.

The $1 \mathrm{E}$ and $2 \mathrm{E}$ sensor performance can compared by looking at the two detectors irradiated to $3 \times 10^{15} \mathrm{n}_{\mathrm{eq}} / \mathrm{cm}^{2}$. They are indicated in blue and red in Fig. 5. Thanks to the shorter inter-electrode distance, the efficiency of the $2 \mathrm{E}$ detector shows a steeper increase as a function of the bias voltage than the 1E one (Fig. 5a). For the same reason, the electric field at the edge of the pixel is more intense for the $2 \mathrm{E}$ device, determining a higher cluster size 2 probability, as shown in Fig. $5 \mathrm{~b}$.

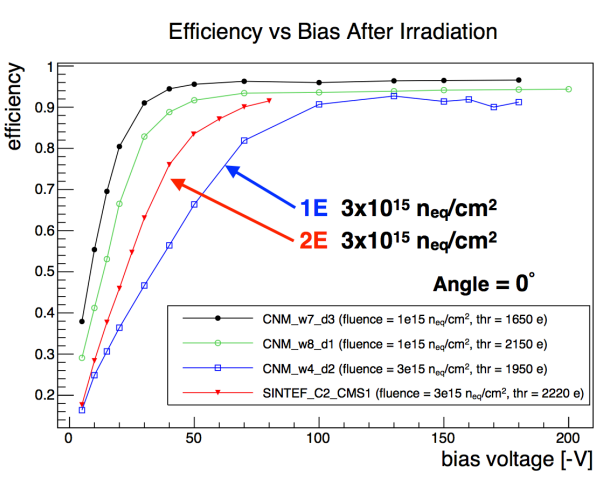

(a) Efficiency vs bias voltage.

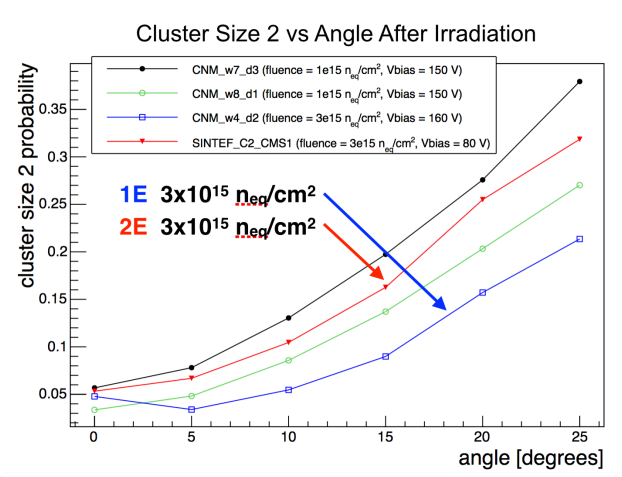

(b) Cluster size 2 probability vs tilting angle.

Figure 5. Efficiency as a function of bias voltage (left) and cluster size 2 probability vs tilting angle (right), after irradiation.

\subsection{CT-PPS module qualification}

Before the installation in the RPs, modules go through a severe qualification procedure. After the current vs voltage (IV) measurement at CNM, wafers are sent to IZM for the bump-bonding of the selected sensors. ROCs are tested on wafer and after dicing, following the standard CMS Phase I procedure. After the delivery from IZM, modules are characterized by means of a temporary wire-bonding and gluing with gel-pak film to the CT-PPS flex readout circuit.

The sensor IV curve is measured in order to check for damages, and ROCs are tested and their parameters optimized. Then data are acquired while irradiating the module with X-rays to verify that all pixels see entries and hence are properly bump-bonded to the ROC. After the module qualification, it is also possible to select its orientation for the final gluing to ensure that possible bad pixels are not placed close to the beam, where higher occupancy is expected. The Genova and Torino laboratories have equivalent setups for the full qualification procedure, which started in both sites with good results so far.

\section{CT-PPS tracking electronics, mechanics and cooling}

The CT-PPS back-end electronics will be identical to the one for the CMS Phase I pixel detector. The interface between that and the modules is composed of two devices: the RPix Port Card and the flex hybrid. There is one RPix Port Card per pot. The card is custom designed starting from the TOTEM Port Card, which fits inside the Roman pot; the components however are those of 
the Phase I pixel detector. The RPix Port Card enables the communication between the back-end electronics and the modules, which are connected to the Port Card by means of the flex hybrids. This latter device is also custom designed for CT-PPS and is able to read both $2 \times 3$ and $2 \times 2$ modules. The RPix Port Card prototype is under test in Genova with good results; final production is foreseen to be launched in October. The first hybrid flex production was completed successfully. The second production is ongoing, with completion expected by the beginning of October.

The flex hybrid is glued on a Thermal Pyrolytic Graphite (TPG) layer that provides the heat dissipation for the pixel module. The TPG is connected to the aluminum structure that hosts the 6 planes of the tracking station together with the cooling system, which is identical to the one of TOTEM strips. The first aluminum structure has already been produced and has been used for cooling tests with very good results. The remaining required structures are in production.

\section{Conclusions}

The CT-PPS tracking system will consist of two stations installed on each side of the CMS experiment at $\sim 210 \mathrm{~m}$ from the IP. Each station will have six planes of 3D pixel detectors. Test results of the CT-PPS prototype detectors match well the project requirements. Mechanics and electronics are under test with good results. Installation is foreseen during the 2016-17 extended winter LHC shutdown.

\section{Acknowledgments}

Special thanks go to the CT-PPS tracking group. We thank the FERMILAB T-992 collaboration for the contribution provided during beam tests. We would also like to express our gratitude to CNM and SINTEF for the production of the sensors that we tested. Finally, we thank the team of the CERN IRRAD Proton Facility for the irradiation of the samples.

\section{References}

[1] The CMS-TOTEM Collaboration, CMS-TOTEM Precision Proton Spectrometer, CERN-LHCC-2014-021 (2014)

[2] The CMS Collaboration, The CMS experiment at the CERN LHC, JINST 3 (2008) S08004

[3] The CMS Collaboration, CMS Technical Design Report for the Pixel Detector Upgrade, CERN-LHCC-2012-016 (2012)

[4] The CMS Collaboration, Search for new physics in high mass diphoton events in proton-proton collisions at 13 TeV, CMS-PAS-EXO-15-004 (2015)

[5] The ATLAS Collaboration, Search for resonances decaying to photon pairs in $3.2 \mathrm{fb}^{-1}$ of $\mathrm{pp}$ collisions at $\sqrt{s}=13 \mathrm{TeV}$ with the ATLAS detector, ATLAS-CONF-2015-081 (2015) 